\title{
Decomposing Components of Task Preparation with Functional Magnetic Resonance Imaging
}

\author{
Marcel Brass and D. Yves von Cramon
}

\begin{abstract}
It is widely acknowledged that the prefrontal cortex plays a major role in cognitive control processes. One important experimental paradigm for investigating such higher order cognitive control is the task-switching paradigm. This paradigm investigates the ability to switch flexibly between different task situations. In this context, it has been found that participants are able to anticipatorily prepare an upcoming task. This ability has been assumed to reflect endogenous cognitive control. However, it is difficult to isolate task preparation process from task execution using functional magnetic resonance imaging (fMRI). In the present study, we introduce a new experimental manipulation to investigate task preparation with fMRI. By manipulating the number of times a task was prepared, we could demonstrate
\end{abstract}

\section{INTRODUCTION}

It is widely acknowledged that the prefrontal cortex plays a major role in the coordination of goal-directed behavior (Miller \& Cohen, 2001; Fuster, 1980; Milner, 1963). One crucial aspect of this coordinative function is our ability to prepare for specific task situations before we encounter the actual task (Sudevan \& Taylor, 1987). Imagine, for example, that you are driving down a road and see a traffic sign signaling that there is a traffic light around the next corner. You will be able to use this sign to prepare for the upcoming task without knowing whether the traffic light will be red or green. This example suggests that we can anticipatorily adjust to a relevant task. In the terminology of cognitive psychology, we are able to prepare the task set that determines the relevant task rules (Rogers \& Monsell, 1995).

Experimentally, task set preparation can be investigated with a cueing version of the so-called task-switching paradigm (Mayr \& Kliegl, 2000; Meiran, 1996; Sudevan \& Taylor, 1987). In this paradigm, participants are required to alternate between two different tasks. Because the task rules change between the tasks, participants constantly have to adjust to the relevant task set. By presenting a task cue in advance, it is possible to temporally dissociate task preparation from task execution.

Max Planck Institute of Cognitive Neuroscience that the left inferior frontal junction (IFJ) area (near the junction of inferior frontal sulcus and inferior precentral sulcus), the right inferior frontal gyrus, and the right intraparietal sulcus are involved in task preparation. By manipulating the cue-task mapping, we could further show that this activation is not related to cue encoding but to the updating of the relevant task representation. Based on these and previous results, we assume that the IFJ area constitutes a functionally separable division of the lateral prefrontal cortex. Finally, our data suggest that task preparation does not differ for switch and repetition trials in paradigms with a high proportion of switch trials, casting doubt on the assumption that an independent task set reconfiguration process takes place in the preparation interval.

In behavioral experiments, it has been demonstrated that switching between tasks involves cognitive control processes that go beyond simple task-specific processing. This cognitive control effort is reflected in so-called switch costs (longer reaction times for switch compared to repetition trials). When participants are able to prepare the next task, reaction time decreases and a reduction of switch costs is observed (Meiran, 1996). It was initially assumed that the reduction of switch costs reflects the advanced reconfiguration of the task set (Rogers \& Monsell, 1995) or the preparation of the stimulus task set (Meiran, 1996). Recently however, alternative models have been proposed, which do not postulate a switch-specific control process to be related to task preparation (Logan \& Bundesen, 2003). While the precise functional role of task preparation is still a matter of controversy, a number of neuroimaging studies have tried to isolate the neuronal mechanisms involved in task preparation (Luks, Simpson, Feiwell, \& Miller, 2002; Rushworth, Paus, \& Sipila, 2001; Kimberg, Aguirre, \& D'Esposito, 2000; MacDonald, Cohen, Stenger, \& Carter, 2000).

\section{Imaging Studies on Task Preparation}

One major problem with using neuroimaging methods to isolate task preparation from task execution is that the 
time needed to prepare a task is very short while the hemodynamic response is relatively slow. This leads to an overlap of the hemodynamic response for the cue and target period. Modeling the cue- and target-related processing with overlapping BOLD signals and nonorthorgonal experimental conditions is possible, but relatively complicated (Toni, Rushworth, \& Passingham, 2001). Accordingly, some authors have used long preparation intervals to temporally separate these processes (Luks et al., 2002; MacDonald et al., 2000). However, this approach has the disadvantage that working memory in the sense of maintenance of information comes into play. Another strategy used to investigate task preparation is to manipulate task-order predictability (Dreher, Koechlin, Ali, \& Grafman, 2002; Kimberg et al., 2000; Sohn, Ursu, Anderson, Stenger, \& Carter, 2000). This approach elaborates on the alternating-runs paradigm (Rogers \& Monsell, 1995). In this paradigm, the order of task presentation allows the next task to be predicted. However, in behavioral studies it was found that task preparation processes might differ for explicit task cueing and predictability of task order (Koch, 2004). Bearing this in mind, it is not surprising that empirical findings on the neuronal basis of task preparation are relatively heterogeneous. Most studies have found preparation-related activation in the prefrontal cortex (Dreher et al., 2002; Luks et al., 2002; MacDonald et al., 2000; Sohn et al., 2000). However, these activations spread over the whole prefrontal cortex. Furthermore, some studies have found a difference between switch and repetition trials in the preparation phase (Kimberg et al., 2000; Sohn et al., 2000) whereas others have not (Luks et al., 2002), casting doubt on the existence of a task reconfiguration process. We have recently chosen a different approach to isolate task preparation from task execution (Brass \& von Cramon, 2002). Using trials in which only a task cue but no task was presented, we were able to dissociate cue- and target-related processing, without confounding working memory processes. When compared to low-level baseline, activation was found in the vicinity of the posterior extent of the inferior frontal sulcus at the junction with the inferior precentral sulcus. We termed this area the inferior frontal junction area (IFJ). Medial frontal activation was found in the pre-SMA. Although this study succeeded in dissociating cue- and target-related brain activation, it left two fundamental questions open. First, our study, like others, did not show any switchspecific differences in the preparation phase, suggesting that there might not be a switch-specific preparation process (Ruge et al., submitted). However, if task preparation does not differ for switch and repetition trials, the switch manipulation is not appropriate for identifying the cortical regions involved in task preparation. Therefore, one aim of the present study was to introduce an experimental paradigm that manipulates task preparation independently from trial transition. Second, previous experiments on explicit task cueing did not address the question of whether the activation in the frontal cortex reflects the coding of the cue or the updating of the relevant task set. To address these two questions, we devised a new paradigm that manipulated the number of times a task was prepared as well as the cue-task mapping.

\section{A Functional Magnetic Resonance Imaging-Related Approach to Investigating Task Preparation}

The rationale behind this paradigm was to use the strength of neuroimaging methods to decompose cognitive processes. In contrast to reaction time research, no response is needed to investigate a mental process. In our paradigm, participants were given two task cues separated by a preparation interval before the target was presented (Figure 1). These task cues indicated either the same or a different task. In addition, catch trials were presented in which the target appeared after the first cue. These catch trials ensured that participants paid attention to the first cue. Furthermore, this single-cue condition could be used to investigate the trial transition effect. For the double-cue conditions, we predicted that if both cues indicate the same task, participants only prepare once, whereas if the second cue indicates a different task they should prepare twice. The second experimental manipulation was related to the question of which specific processes are reflected by cue-related activation. By assigning two cues to each task, we can compare a switch of both cues without a switch of cue meaning (two different cues that indicate the same task) with a switch of cues and cue meaning (two cues that indicate different tasks) (Logan \& Bundesen, 2003; Mayr \& Kliegl, 2003; Bunge, Kahn, Wallis, Miller, \& Wagner, 2003). While the coding of the cue is required in both conditions, participants are only required to prepare the task twice in the condition in which the cue meaning change. In addition to trial type, we manipulated the cue-target interval (CTI). In the short CTI, participants were required to respond $60 \mathrm{msec}$ after the relevant cue (the second cue in the three double-cue conditions and the first cue in the single-cue condition), while they had 700 msec to prepare in the long CTI condition. The CTI manipulation was introduced as an additional manipulation check to ensure that the first cue was indeed processed. If the double-cue conditions differ regarding the processes involved, we can expect a reaction time difference in the short CTI condition, but not in the long CTI condition. Hence, the experimental design consisted of the factors trial type (cue repetition, cue switch, meaning switch) and CTI (short, long). The single-cue condition was analyzed separately because of the different trial structure. 


\section{RESULTS}

\section{Behavioral Data}

The response time data were analyzed using a repeated measurement ANOVA with the factors trial type and CTI (Figure $2 \mathrm{~A}$ ). A main effect of trial type, $F(2,26)=25$, $p<.01$, and CTI, $F(1,13)=283, p<.01$, was found. Most interestingly, the interaction was also significant, $F(2,26)=7, p<.01$. In the short CTI condition, participants were significantly faster when the cue was repeated than when it was switched, $t(13)=3.7, p<.01$. Furthermore, a significant reaction time difference was found between the meaning-switch and the cue-switch condition, $t(13)=3.5, p<.01$ ). These data suggest that participants indeed used the first cue to prepare the task. Otherwise, no reaction time difference would have been expected between the three types of trials. In the long CTI condition, the only significant reaction time difference was found between the meaning-switch condition and the other conditions, $t(13)=2.3, p<.05$ (Figure 2B). This result indicates that even in the long CTI, participants are not able to completely overrule the task set activation caused by the first cue.

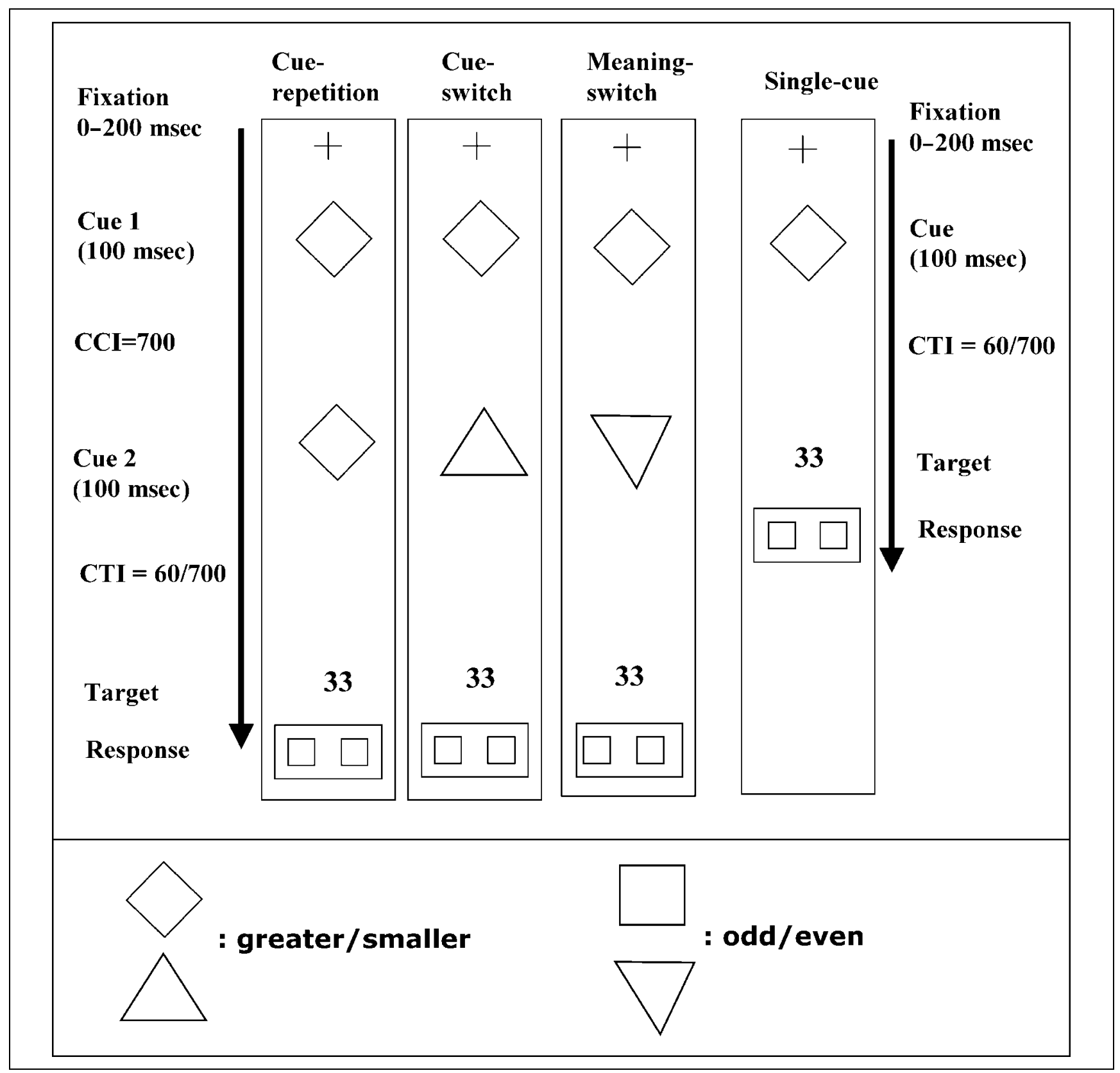

Figure 1. Trial structure and exact experimental timing. In the three double-cue conditions, two task cues were presented before the target was displayed. In the single-cue condition, the target was presented after the first cue. The lower part of the figure displays the cue-task mapping. Two different cues were assigned to each task. 


\section{cue-transition for double-cue trials}

A

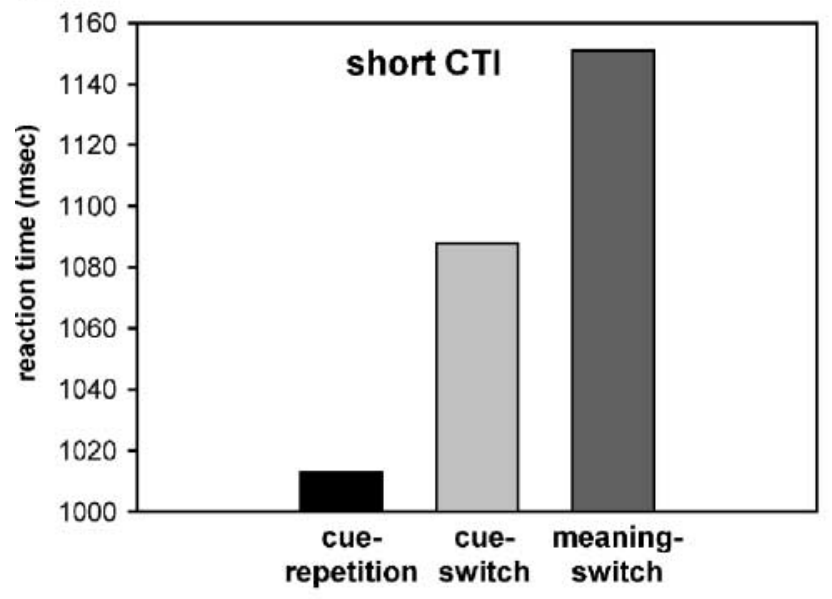

B

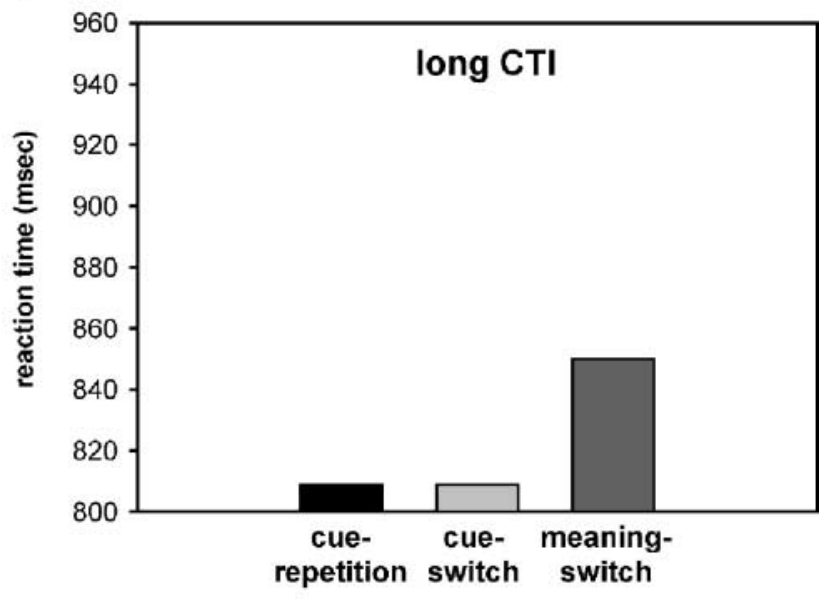

trial-transition for single-cue trials

C

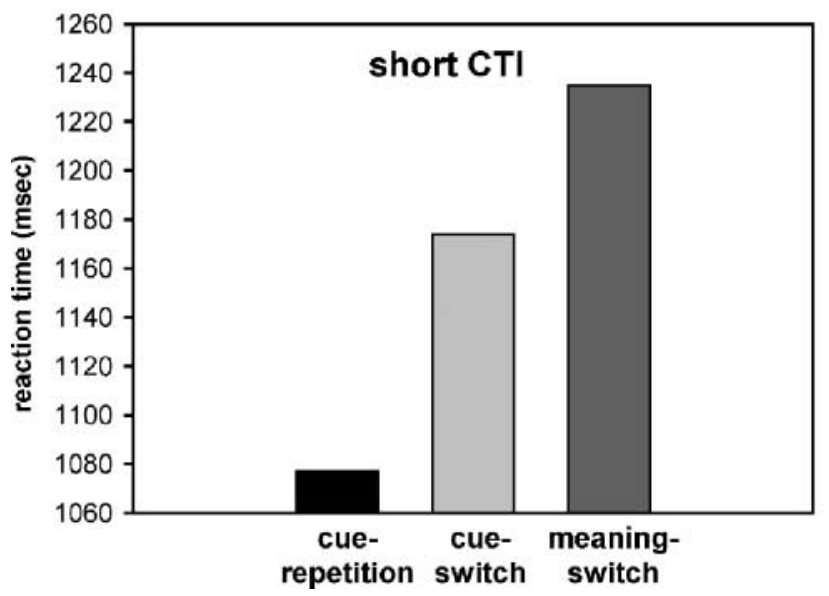

D

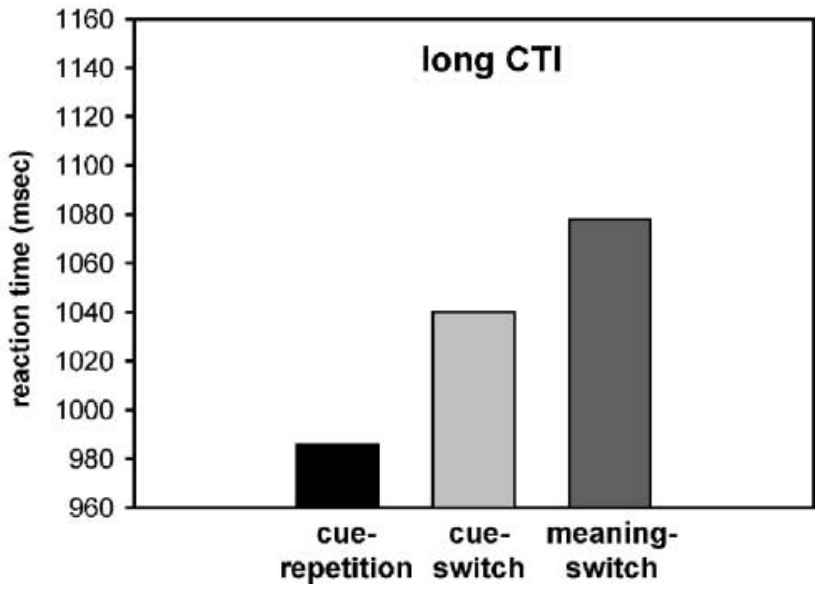

Figure 2. (A) Reaction time as a function of cue-transition in the short CTI condition (double-cue conditions). (B) Reaction time as a function of cue transition in the long CTI condition (double-cue conditions). (C) Reaction time as a function of trial transition in the short CTI (single-cue conditions). (D) Reaction time as a function of trial transition in the long CTI (single-cue conditions).

The comparison of short and long CTI in the singlecue condition revealed the expected strong preparation effect of about $130 \mathrm{msec}, t(13)=12.0, p<.01$, also indicating that participants used the first cue to prepare the task. Furthermore, we computed the trial-transition effects for the single-cue condition (Figure 2C and D). This analysis was carried out by comparing the last cue in trial $n-1$ with the cue in trial $n$. Equivalent to the within-trial manipulation one can distinguish three types of between-trial transitions: a switch in cue meaning, a cue switch, and a cue repetition. A main effect for transition type was found, $F(2,12)=31,88, p<.01$. Participants were faster when the cue in trial $n-1$ was identical to the cue in trial $n$ compared to the condition in which the cue switched, $t(13)=5.6, p<.01$. Furthermore, subjects were faster when the cue but not the cue-meaning changed, $t(13)=2.8, p<.05$. No interaction of CTI and transition type was found.

\section{Functional Magnetic Resonance Imaging Data}

For the functional magnetic resonance imaging (fMRI) analysis, we pooled both CTI conditions, since the CTI manipulation was only introduced as a manipulation check for the first cue and we know from previous experiments (Brass \& von Cramon, 2002) that it has no effect on the preparation-related activation. The crucial contrast was the difference between the meaning-switch condition and the cue-switch condition (see Figure 3 and Table 1). Since both conditions involve a cue-switch, this contrast should indicate cortical regions that are related to the updating of the relevant task set, 


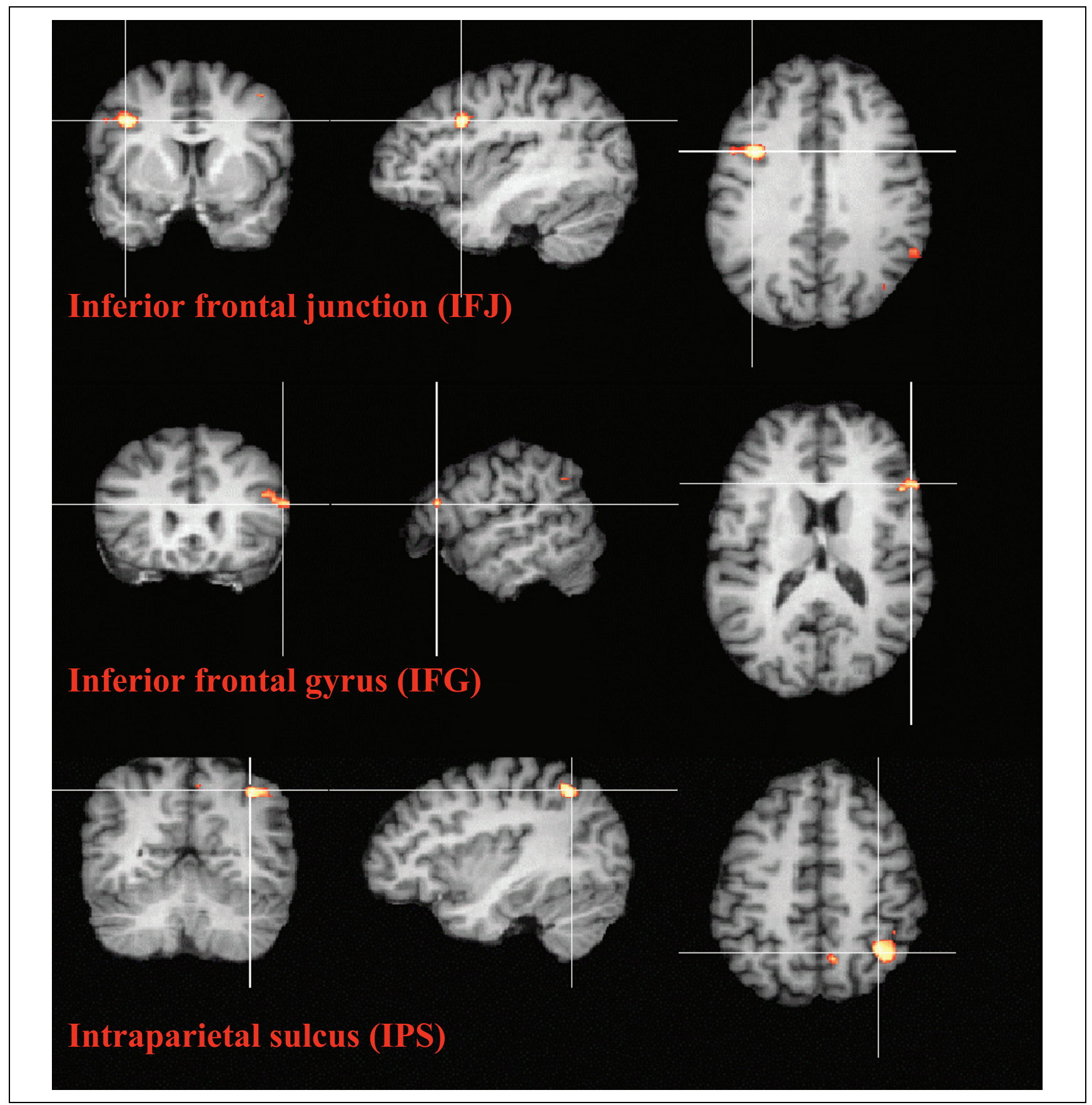

Figure 3. Cortical activation for the contrast of meaning switch versus cue switch with a $z$ value $>3.1$.

but not to the coding of the cue. In accordance with our hypothesis, this contrast yielded strong activation in the left IFJ. This activation was only a few millimeters anterior to the fronto-lateral activation we found in our previous study on task preparation (Brass \& von Cramon, 2002). In addition, activation was found in the right inferior frontal gyrus (IFG). Finally, the horizontal branch of the right intraparietal sulcus (IPS) was found to be activated. No significant activation was found in the pre-SMA. To check whether the pre-SMA was activated when compared to baseline, we carried out a signal change analysis with the mean coordinate $(x=1$, $y=5, z=53$ ) from Brass and von Cramon (2002), which indicated cue-related activation (Figure 4, last panel). Interestingly, a significant activation of the pre-SMA was found in all three conditions, while no activation difference was found between the meaning-switch and the cue-switch condition, $t(13)=.91, p=.37$. However, the cue-repeat condition was significantly less activated, $t(13)=2.6, p<.05$.

The second relevant contrast was related to the comparison of the cue-switch condition and the cue- 
Table 1. Cortical Activation for the Comparison of Meaning Switch versus Cue Switch with a $z>3.1$ and a Minimum Volume Size of $225 \mathrm{~mm}^{3}$ (Five Adjacent Voxels)

\begin{tabular}{lcccc}
\hline & Size $\left(\mathrm{mm}^{3}\right)$ & $z$ Max & Laterality & Talairach Coordinates \\
\hline IFJ & 976 & 4.13 & Left & $-37,5,32$ \\
IFG & 770 & 3.17 & Right & $55,20,18$ \\
IFS & 935 & 4.01 & Right & $35,-58,44$ \\
\hline
\end{tabular}

repetition condition. This contrast should indicate whether the prefrontal cortex was also involved when the cue, but not the cue-meaning, changed. No significant activation was found with the $z$ threshold of 3.1. To investigate whether there might be some subthreshold activation in the prefrontal cortex, we lowered the $z$ threshold to 2.6. However, even with this lower threshold, no prefrontal areas were found to be activated. Rather, activation was found in the lateral premotor cortex, the inferior temporal gyrus, and the fusiform gyrus (Table 2). This activation indicated the encoding of the cue information.

The signal changes indicate that the IFJ, the right IFG, and the IPS were activated in all three experimental conditions when compared to baseline (Figure 4). However, it was only when the cue-meaning changed that this activation increased compared to the cuerepetition condition, $t(13)=4.0, p<.01$. No significant difference was found for the comparison of the cueswitch and cue-repetition conditions, $t(13)=.83$, $p=.41$. Finally, we wanted to investigate whether the cortical areas that showed a significant activation difference in the meaning-switch versus cue-switch contrast were also found to be activated when we compare switch versus repeat trials in the single-cue condition. We restricted the trial transition analysis to the singlecue condition, because trial transition is not unambiguously defined for all double-cue conditions. From previous research, we know that the transition effect is sensitive to the CTI manipulation (Ruge et al., submitted). Therefore, we analyzed the transition effect
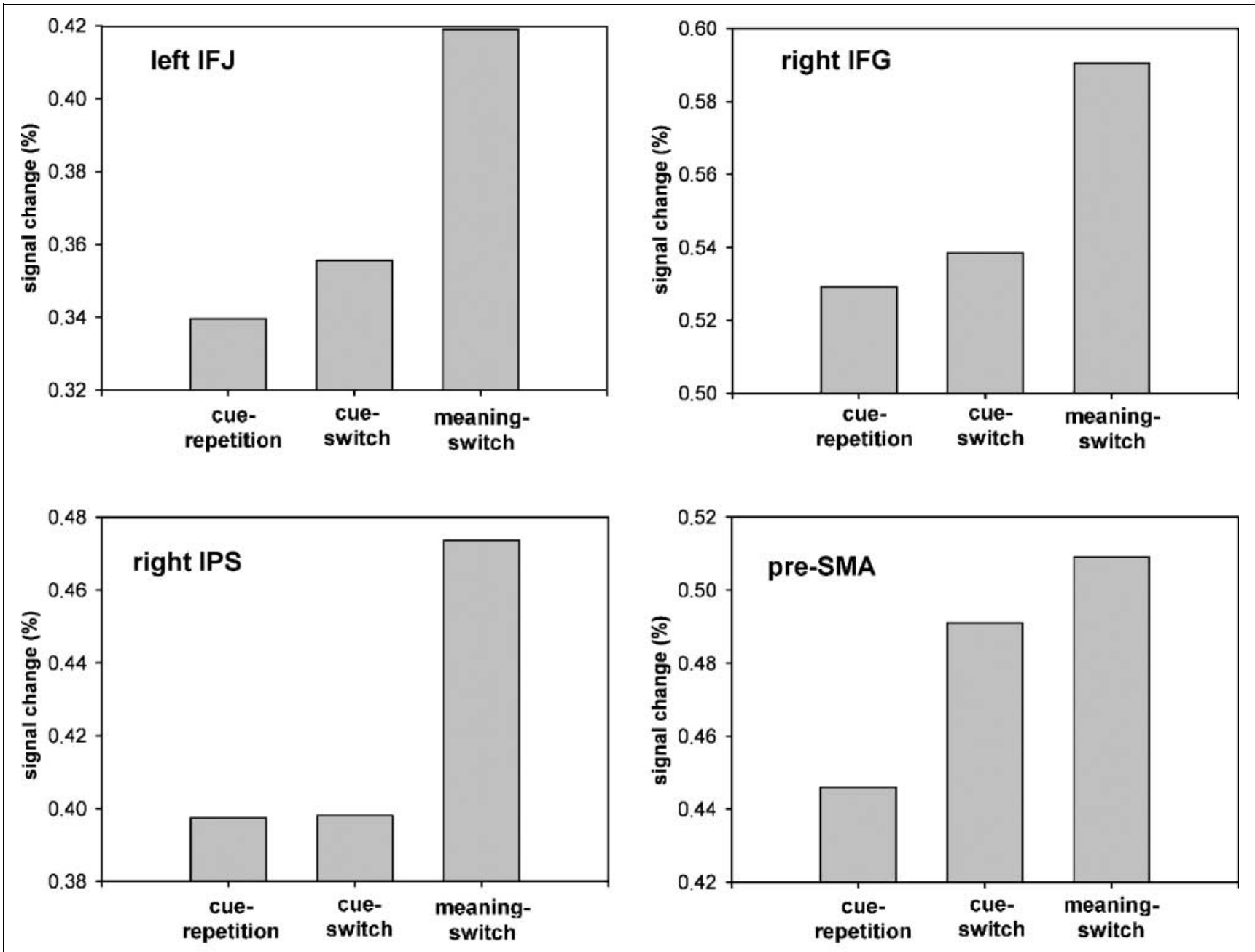

Figure 4. Signal change diagrams for the three most activated cortical areas in the meaning-switch versus cue-switch contrast. The signal changes of the pre-SMA were computed in the coordinate $(x=1, y=5, z=53)$ taken from Brass and von Cramon (2002). 
Table 2. Cortical Activation for the Comparison of Cue Switch versus Cue Repetition Trials with a $z>2.6$ and a Minimum Volume Size of $225 \mathrm{~mm}^{3}$ (Five Adjacent Voxels)

\begin{tabular}{lcccc}
\hline & Size $\left(\mathrm{mm}^{3}\right)$ & z Max & Laterality & Talairach Coordinates \\
\hline Lateral premotor cortex & 371 & 3.55 & Left & $-40,-4,47$ \\
Fusiform gyrus/inferior temporal gyrus & 439 & 3.13 & Right & $49,-55,-9$ \\
Fusiform gyrus/inferior temporal gyrus & 428 & 3.52 & Left & $-49,-58,-9$ \\
Fusiform gyrus & 655 & 3.55 & Right & $32,-79,-3$ \\
\hline
\end{tabular}

for both CTI conditions separately. Because of the small trial number, we restricted the analysis to the comparison of switch and repetition trials without further distinguishing between the cue-switch and the cuerepetition condition. Furthermore, we again lowered the $z$ threshold to 2.6 since we had a clear a priori hypothesis about the relevant brain areas. In accordance with previous results, a switch-specific activation was found for the left IFJ, the pre-SMA, and the dorsal premotor cortex in the short CTI condition, but not in the long CTI condition (Table 3).

\section{DISCUSSION}

The aim of the present study was twofold. First, we wanted to introduce an experimental manipulation that varied the task preparation processes independently from trial transition. Second, we wanted to dissociate cortical regions related to the encoding of the cue from those related to the updating of the task set. The findings of the present study are clear-cut in both respects. The behavioral data demonstrate that task preparation can be manipulated within an experimental trial. In the short CTI condition, participants were fastest when both the cue and the cue meaning were repeated. When the cue changed but the cue meaning was identical, there was an increase in reaction time. Most importantly, reaction times were slowest when both the cue and the cue meaning changed. These data strongly suggest that two different cognitive processes can be dissociated. This assumption is further supported by the analysis of the trial-transition effects, which showed a similar result. One process is related to cue encoding. When the same cue was presented twice participants only had to code it once, whereas when the cue switched, they had to code the cue twice. In the
fMRI data, this process was reflected in the activation of the fusiform gyrus/inferior temporal cortex and the lateral premotor cortex. These activation foci were in accordance with existing literature. The fusiform gyrus is known to be related to the visual processing of objects (Rao, Zhou, Zhou, Fan, \& Chen, 2003; Kanwisher, Woods, Iacoboni, \& Mazziotta, 1997). Furthermore, a recent study by Schumacher and D'Esposito (2002) reported a dissociation of extrastriate visual areas and a fronto-parietal network related to stimulus encoding and response selection. The ventral premotor cortex has been shown to be involved in the sensory processing of objects (Schubotz \& von Cramon, 2002). We assume that the premotor cortex activation reflects the pragmatic features of the attended cue (Schubotz \& von Cramon, 2002; Fadiga, Fogassi, Gallese, \& Rizzolatti, 2000).

More importantly, the reaction time difference between the cue-switch and meaning-switch condition indicated that the processing of cue meaning also led to a significant reaction time increase. This experimental variation was related to the IFJ, the right IFG, and the IPS. While the activation in the left IFJ and the right IPS replicate our previous findings, which suggest that these regions are involved in task preparation, the right IFG was not found to be activated in our previous study on task preparation (Brass \& von Cramon, 2002).

In the following paragraphs, we will discuss the functional mechanisms that might be behind the functional role of these activation.

\section{Task Preparation: What Does It Mean?}

The present findings extend the results of our previous study (Brass \& von Cramon, 2002), which could demonstrate cue-related activation in the prefrontal cortex

Table 3. Cortical Activation for the Contrast of Switch and Repetition Trials in the Short CTI of the Single Cue Condition with a $z>2.6$ and a Minimum Volume Size of $225 \mathrm{~mm}^{3}$ (Five Adjacent Voxels)

\begin{tabular}{lcccc}
\hline & Size $\left(\mathrm{mm}^{3}\right)$ & $z$ Max & Laterality & Talairach Coordinates \\
\hline Pre-SMA & 1468 & 3.96 & Left & $-5,2,50$ \\
Lateral premotor cortex & 725 & 3.66 & Left & $-23,-10,50$ \\
Inferior frontal junction & 761 & 3.58 & Left & $-37,8,35$ \\
\hline
\end{tabular}


but could not decide whether this activation was related to the encoding of the cue or to the updating of the task set. The present data clearly indicate prefrontal involvement in task preparation processes that go beyond simple cue encoding. More specifically, we assume that the left IFJ is involved in the processing of the relevant task set. Rubinstein, Meyer, and Evans (2001) recently dissociated two subcomponents related to task switching, namely, goal shifting and rule activation. While goal shifting is related to the activation of a general task representation, the rule activation process is responsible for activating task-specific stimulus-response rules. Goschke (2000) proposed a concept that is similar to the goal-shifting component. He argued that task preparation mainly requires the retrieval of the relevant task representation. Based on the present experiment, we cannot dissociate the updating of the general task representation, from the activation of specific stimulusresponse rules. By showing that prefrontal neurons represent abstract task rules (Wallis, Anderson, \& Miller, 2001), recent neurophysiological data support the assumption that the lateral prefrontal cortex is involved in the processing of task representations. However, neurophysiological findings could also show that the lateral prefrontal cortex is involved in the processing of a specific kind of stimulus-response rules, so-called arbitrary motor mappings (Wise \& Murray, 2000; Rushworth \& Owen, 1998). In arbitrary motor mappings, the application of an abstract rule is required to guide behavior. Recently, Bunge et al. (2003) directly compared the processing of arbitrary motor mappings and abstract task rules and found stronger activation in a region close to the IFJ for the abstract task representation compared to the arbitrary motor mapping.

In neuropsychology, the idea that the prefrontal cortex is involved in the processing of general task representations (here called goals) was elaborated by Duncan, Emslie, Williams, Johnson, and Freer (1996). They assumed that a major problem of prefrontal patients is what they called goal neglect. Patients with goal neglect have problems representing and flexibly switching between different task goals. Goschke (2000) assumed that the updating of general task representations mainly consists in verbalizing such representations. Interestingly, goal neglect can be dissociated from a failure to verbalize the instruction. Prefrontal patients are often able to report what they should do, but they nevertheless not take the necessary action. This finding suggests that the updating of a task representation goes beyond a simple verbalization strategy.

Regarding the existing imaging literature, the present results are consistent with a number of task-switching studies (Konishi et al., 2002; Dove, Pollmann, Schubert, Wiggins, \& von Cramon, 2000; Pollmann, Dove, von Cramon, \& Wiggins, 2000) and Wisconsin Card Sorting Test (WCST) studies (Nakahara, Hayashi, Konishi, \& Miyashita, 2002; Monchi, Petrides, Petre, Worsley, \&
Dagher, 2001; Nagahama et al., 2001), which have demonstrated the role of the IFJ in cognitive control processes. However, as suggested above, we think that the present study further clarifies the functional role of this cortical region. While more anterior prefrontal regions in the mid-dorsolateral and ventrolateral prefrontal cortex might be related to working memory in the sense of maintenance and manipulation of working memory content (Henson \& Fletcher, 2002; D'Esposito et al., 1998), the IFJ is involved in context-related updating of the general task representation. In this sense, the IFJ constitutes a third, functionally distinct region in the lateral prefrontal cortex. From the neuroanatomical perspective, it makes perfect sense that the IFJ should have a separate role. Based on its cytoarchitectonic structure, Petrides and Pandya (1999) separated the mid-dorsolateral prefrontal cortex from the posterior prefrontal cortex. While the mid-dorsolateral prefrontal cortex is composed of Brodmann's areas (BA) 46 and 9/46, the IFJ is cytoarchitectonically located at the junction of prefrontal area $8 \mathrm{Av}$, premotor area 6 , and the premotor/prefrontal transition cortex BA 44. This makes it perfectly suited to integrate premotor, prefrontal, and verbal information.

\section{Parietal Contributions to Cognitive Control}

The discussion thus far has focused on the role of the left IFJ in task preparation. However, the parietal cortex showed a very similar activational pattern. Furthermore, we found exactly the same IPS activation in our previous study on task preparation (Brass \& von Cramon, 2002). This raises the question as to the functional role of the parietal cortex in cognitive control. Regarding the interaction of prefrontal and parietal cortex, it has been suggested that the parietal cortex receives biasing signals from the prefrontal cortex that influence task-specific processing (Tomita, Ohbayashi, Nakahara, Hasegawa, \& Mijashita, 1999). Bunge, Hazeltine, Scanlon, Rosen, and Gabrieli (2002) recently dissociated different functional roles of prefrontal and parietal cortex in an interference paradigm. While the prefrontal cortex was involved in the selection of the relevant response, the parietal cortex was associated with the representation of candidate responses. Using a task-switching paradigm, Rushworth et al. (2001) found an association of the medial IPS with the motor attention required to initiate intentional switches between visuomotor transformation rules. More generally, Corbetta and Shulman (2002) argued that parts of the IPS are involved in the selection of stimulus and response. Based on these results, it is reasonable to postulate a functional dissociation of the parietal and the prefrontal cortex in task preparation. While the IFJ is involved in the updating of the general task representation, the parietal cortex might provide the relevant stimulus-response associations that are needed to execute the task. 


\section{Right Inferior Frontal Gyrus and the Inhibition of Task Representations}

The right IFG was the only cortical region that was not found to be related to task preparation in our previous study (Brass \& von Cramon, 2002). Therefore, we assume that this activation is not related to task preparation per se, but might result from two different task sets competing in working memory when the cue meaning changes. The representation of the first cue has to be overwritten to establish the task representation of the second cue. Interestingly, an activation of the right IFG was also found in a task-switching study that manipulated foreknowledge (Sohn et al., 2000). Here, the activation was stronger in switch than in repetition trials. Compared to a cueing paradigm in which the task instruction is provided before each trial, in the study conducted by Sohn et al. (2000), the order of task presentation indicated which task to execute. In switch trials, the task instruction for trial $n$ has to be retrieved while trial $n-1$ is still active in working memory. The right IFG might be involved in the selection of the second task set when interference occurs from the first task set. This would be consistent with the role of the IFG in selective memory retrieval (for an overview, see Henson \& Fletcher, 2002). It is important to note that we expect such interference effects only if two task sets have to be held in a preparatory state. As soon as the participant has executed one task, the respective task set will be discarded from working memory. This is why such interference effects usually do not occur for the transition between trials (see below).

\section{Pre-SMA and Task Preparation}

Another puzzling aspect of the present results was the lack of significant activation in the pre-SMA for the whole brain analysis. In previous studies, the pre-SMA has been found to be involved in task switching (Rushworth, Hadland, Paus, \& Sipila, 2001; Dove et al., 2000). Furthermore, it has been shown that the pre-SMA is involved in cue-related processing (Brass \& von Cramon, 2002; Luks et al., 2002). Based on these results, we expected to find an activation difference for the meaning-switch versus cue-switch contrast. However, such an effect was not found. Even if the signal change analysis indicated that the pre-SMA was significantly activated in all three conditions, a significant decrease in activation was only found in the cue repetition condition. In other words, this cortical region is only sensitive to a change in the cue but not in the cue meaning. In contrast to our previous assumption (Brass \& von Cramon, 2002), the present data indicate that the pre-SMA is not related to task-specific preparation. Rather, we would suggest that the pre-SMA activation reflects some kind of general preparedness in situations in which the relevant re- sponses are uncertain. This would be consistent with the role of the pre-SMA in response competition (Garavan, Ross, Murphy, Roche, \& Stein, 2002; Ullsperger \& von Cramon, 2001). Another interpretation of the preSMA might be that this region is sensitive to a cue change because a cue change might indicate a change of behavioral strategy.

\section{Task Preparation and Task Set Reconfiguration}

Unlike most other studies, we have identified the cortical regions that are related to task preparation by manipulating the number of times a task was prepared and not by comparing switch and repetition trials. The question arises of whether our manipulation activated the same prefrontal regions as the manipulation of trial transition. When analyzing the trial transition effect in the single-cue condition, we found a significant switch effect in the IFJ and the pre-SMA in the short CTI condition, whereas no prefrontal area was activated in the long CTI condition. These data suggest that the left IFJ is sensitive to both the manipulation of trial transition and the manipulation of task preparation. Based on these results, one might argue that the IFJ is involved in anticipatory task set reconfiguration. However, such an interpretation is not consistent with the finding that this region was only activated in the short CTI condition and not in the long CTI condition. If the activation difference between switch and repetition trials takes place in the preparation phase, we would not expect any difference of the switch effect between the long and short CTI condition. Based on these data and previous findings of no switch-specific activation difference in the preparation phase (Brass \& von Cramon, 2002; Luks et al., 2002), we assume that the IFJ is involved in task preparation, but that this task preparation process does not differ for switch and repetition trials in the preparation phase. In an experimental context in which there is an equal likelihood of switch and repetition trials, which is indeed the case in most task switching studies, participants tend to prepare the task in each trial. Therefore, prefrontal brain regions do not show a consistent switching effect. Interestingly, studies on the WCST consistently found posterior prefrontal activation (Nakahara et al., 2002; Monchi et al., 2001; Nagahama et al., 2001). Most of these studies use a simplified version of the WCST that is very similar to a task-switching paradigm. In WCST studies, however, the likelihood of switch trials is usually much smaller than $50 \%$. Therefore, subjects in WCST paradigms are less required to prepare the task set in repetition trials, leading to stronger switch effects.

But still the question of why we found a switch effect in the short CTI condition but not in the long CTI remains open. Based on another study, in which we directly addressed this question, Ruge et al. (submitted) assumed that the switch effect in the short CTI condition 
does not reflect task set reconfiguration but results from the higher control effort required when the task is presented before the task representation is specified. In switch trials, interference arises because the experimental stimulus activates the previous task set (Allport, Styles, \& Hsieh, 1994) and the task representation has to be strengthened to overcome this task incongruent activation. Such an interpretation would suggest that the updating of task representations can be carried out anticipatorily to prepare the task set but also after stimulus presentation to overcome task set inertia.

\section{Conclusions}

In the present experiment, we dissociated two cognitive components related to the processing of task cues. One component, which is located in the fusiform gyrus and the dorsal premotor cortex, is related to cue encoding. The second component is located in the left IFJ, the right IFG, and the IPS. Based on this study and a previous study, we assume that the left IFJ is responsible for activating a general task representation, whereas the IPS might be involved in providing the relevant stimulus-response associations. The right IFG seems to be related to the selective retrieval of the relevant task set when interference arises from a nonrelevant task set. The pre-SMA, which was previously assumed to be related to task preparation, showed no specific response to the manipulation of cue meaning, indicating that it is involved in a task-unspecific process of general preparedness. Furthermore, we assume that in a taskswitching paradigm with an equal likelihood of switch and repetition trials, the updating of the task representation takes place in each experimental trial, regardless of whether the trial is a switch or repeat trial. Therefore, it is difficult to identify preparation-related activation by manipulating trial transition. Finally, our data demonstrate how the specific advantages of neuroimaging (manipulating mental processes independently from the response) can contribute to the understanding of cognitive processes.

\section{METHODS}

\section{Experimental Design}

In this study, we used a modified version of the paradigm introduced by Sudevan and Taylor (1987). Digits between 20 and 40 (except 30) were presented on the computer screen. Participants had to execute two tasks: judging whether a digit was smaller or greater than 30 (magnitude task) and judging whether the digit was odd or even (parity task). Which task they had to execute was signaled by a task cue presented as a frame surrounding the digit. In the double-cue conditions, participants received two task cues, before the actual task was presented. These task cues could indicate the same or a different task. The trial length was $6 \mathrm{sec}$. Trials started with a variable oversampling interval of $0,500,1000$, or $1500 \mathrm{msec}$ to obtain a temporal resolution of $500 \mathrm{msec}$. The experimental trial began with a fixation cross that was presented for $200 \mathrm{msec}$. The first task cue was then presented for $100 \mathrm{msec}$. Before the presentation of the second cue, a fixed intercue interval of $700 \mathrm{msec}$ was inserted. After the second cue and a CTI of 60 or $700 \mathrm{msec}$, the target was presented for $400 \mathrm{msec}$. Participants had 2000 msec to respond to the target. After the response window, a feedback was displayed for 200 msec. In single-cue trials, the task was presented 60 or 700 msec after the first cue. Two different task cues were assigned to each task (triangle and diamond to the parity task and square and inverted triangle to the magnitude task). This resulted in three double-cue conditions: a condition in which both the cue and the cue meaning were repeated (cue repetition condition), a condition in which the cue switched, but both cues indicated the same task (cue-switch condition) and a condition in which the cue switched to indicate the other task (meaning-switch condition).

The experiment consisted of two blocks, with 192 trials each. One hundred ninety-two double-cue trials (64 cue repetition/64 cue switch/64 meaning switch) were randomly intermixed with 128 single-cue trials and 64 null events.

\section{Participants}

Fourteen participants ( 8 women and 6 men) who gave informed consent participated in the present study. All participants (mean age: 24.4 years, standard deviation: 1.9) were right-handed as assessed by a German adaptation of the Edinburgh Handedness Inventory (Oldfield, 1971) and had no neurological abnormalities.

\section{Functional Magnetic Resonance Imaging Analysis}

The experiment was carried out on a 3-T scanner (Medspec 30/100, Bruker, Ettlingen). Twenty axial slices $(19.2 \mathrm{~cm}$ FOV , $64 \times 64$ matrix, $4 \mathrm{~mm}$ thickness, $1 \mathrm{~mm}$ spacing), parallel to the AC-PC plane, and covering the whole brain, were acquired using a single shot, gradientrecalled EPI sequence (TR $2000 \mathrm{msec}$, TE $30 \mathrm{msec}, 90$ flip angle). Prior to the functional runs, 20 corresponding anatomical MDEFT slices and 20 EPI-T1 slices were acquired. Stimuli were presented using a headmounted display with a resolution of $1024 \times 768$ and a refresh rate of $60 \mathrm{~Hz}$.

Analysis of fMRI data was performed using the LIPSIA software package (Lohmann et al., 2001). First, functional data were corrected for movement artifacts. The temporal offset between the slices acquired in one scan were then corrected using a sync interpolation algorithm. Data were filtered using a spatial Gaussian filter with sigma $=1.0$. A temporal highpass filter with a cutoff frequency of $1 / 100 \mathrm{~Hz}$ was used for baseline correction 
of the signal. In addition, a global scaling was carried out. All functional data sets were individually registered into 3-D space using the participants' individual highresolution anatomical images. This 3-D reference data set was acquired for each participant during a previous scanning session. The 2-D anatomical MDEFT slices, geometrically aligned with the functional slices, were used to compute a transformation matrix containing rotational and translational parameter, which register the anatomical slices with the 3-D reference $\mathrm{T} 1$ data set. These transformation matrices were normalized to the standard Talairach brain size (Talairach \& Tournoux, 1988) by linear scaling and finally applied to the individual functional data. The statistical evaluation was carried out using the general linear model for serially autocorrelated observations (Friston et al., 1995). The design matrix for event-related analysis was created using a model of the hemodynamic response with a variable delay. The model equation was convolved with a Gaussian kernel with a dispersion of 4-sec full width half maximum. Contrast maps were generated for each participant. As the individual functional datasets were all aligned to the same stereotactic reference space, a group analysis was then performed. A one-sample $t$ test of contrast maps across participants (random effects model) was computed to indicate whether observed differences between conditions were significantly different from zero. Subsequently, $t$ values were transformed into $z$ scores. To protect against false positive activations, only regions with a $z$ score higher than 3.1 (for some contrasts, $z>2.6$ was chosen, as described in the Results section) and a minimum volume size of five adjacent voxels were reported.

To compute the signal change, we determined the most activated voxel of the relevant contrast in the mean $z$ map. From this voxel, we extracted the time course of the signal for each participant. We then subtracted the time course of the null event from the time course of the relevant conditions to attenuate the overlap of the BOLD response. We determined the percent signal change to be the largest value in the time window between 4 and 6 sec after cue presentation.

\section{Acknowledgments}

We thank Thomas Arnold for the improvements of the fMRI analysis software. Furthermore, we thank Martina Weber for carrying out the programming and behavioral pretesting of the experiment. Finally, we thank Susannah Goss for languagerelated improvements of the manuscript.

Reprint requests should be sent to Marcel Brass, Max Planck Institute for Human Cognitive and Brain Sciences, Stephanstrasse 1A, D-04103 Leipzig, Germany, or via e-mail: brass@cns. mpg.de.

The data reported in this experiment have been deposited in the fMRI Data Center (http://www.fmridc.org). The accession number is $2-2003-1145 \mathrm{~K}$.

\section{REFERENCES}

Allport, A., Styles, E. A., \& Hsieh S. (1994). Shifting intentional set: Exploring the dynamic control of tasks. In C. Umilta \& M. Moscovitch (Eds.), Attention and performance XV (pp. 421-452). Hillsdale, NJ: Erlbaum.

Brass, M., \& von Cramon, D. Y. (2002). The role of the frontal cortex in task preparation. Cerebral Cortex, 12, 908-914.

Bunge, S. A., Hazeltine, E., Scanlon, M. D., Rosen, A. C., \& Gabrieli, J. D. E. (2002). Dissociable contribution of prefrontal and parietal cortices to response selection. Neuroimage, 17, 1562-1571.

Bunge, S. A., Kahn, I., Wallis, J. D., Miller, E. K., \& Wagner, A. D. (2003). Neural circuits subserving the retrieval and maintenance of abstract rules. Journal of Neurophysiology, 90, 3419-3428.

Corbetta, M., \& Shulman, G. L. (2002). Control of goal-directed and stimulus-driven attention in the brain. Nature Reviews Neuroscience, 3, 201-215.

D'Esposito, M., Aguirre, G. K., Zarahn, E., Ballard, D., Shin, R. K., \& Lease, J. (1998). Functional MRI studies of spatial and nonspatial working memory. Cognitive Brain Research, 7, 1-13.

Dove, A., Pollmann, S., Schubert, T., Wiggins, C. J., \& von Cramon, D. Y. (2000). Prefrontal cortex activation in task switching: An event-related fMRI study. Cognitive Brain Research, 9, 103-109.

Dreher, J. C., Koechlin, E., Ali, S. O., \& Grafman, J. (2002). The roles of timing and task order during task switching. Neuroimage, 17, 95-109.

Duncan, J., Emslie, H., Williams, P., Johnson, R., \& Freer, C. (1996). Intelligence and the frontal lobe: The organization of goal-directed behavior. Cognitive Psychology, 30, 257-303.

Fadiga, L., Fogassi, L., Gallese, V., \& Rizzolatti, G. (2000). Visuomotor neurons: Ambiguity of the discharge or 'motor' perception? International Journal of Psychophysiology, 35, $165-177$.

Friston, K., Holmes, A. P., Worsley, K. J., Poline, J. P., Frith, C. D., \& Frackowiak, R. S. J. (1995). Statistical parametric maps in functional imaging: A general linear approach. Human Brain Mapping, 2, 189-210.

Fuster, J. (1980). The prefrontal cortex: Anatomy, physiology, and neuropsychology of the frontal lobe. New York: Raven.

Garavan, H., Ross, T. J., Murphy, K., Roche, R. A. P., \& Stein, E. A. (2002). Dissociable executive functions in the dynamic control of behavior: Inhibition, error detection, and correction. Neuroimage, 17, 1820-1829.

Goschke, T. (2000). Intentional reconfiguration and voluntary persistence in task set switching. In S. Monsell \& J. Driver (Eds.), Control of cognitive processes: Attention and performance XVIII (pp. 331-355). Cambridge: MIT Press.

Henson, P. C., \& Fletcher, R. N. (2002). Frontal lobes and human memory: Insights from neuroimaging. Brain, 124, 849-881.

Kanwisher, N., Woods, R. P., Iacoboni, M., \& Mazziotta, J. C. (1997). A locus in human extrastriate cortex for visual shape analysis. Journal of Cognitive Neuroscience, 9, 133-142.

Kimberg, D. Y., Aguirre, G. K., \& D'Esposito, M. (2000). Modulation of task-related neural activity in task-switching: An fMRI study. Cognitive Brain Research, 10, 189-196.

Koch, I. (2004). The role of external cues for endogenous advance reconfiguration in task switching. Psychonomic Bulletin and Review, 10(2), 488-492.

Konishi, S., Hayashi, T., Uchida, I., Kikyo, H., Takahashi, E., \& Miyashita, Y. (2002). Hemispheric asymmetry in human lateral prefrontal cortex during cognitive set shifting. Proceedings of the National Academy of Sciences, U.S.A., 9, 7803-7808. 
Logan, G. D., \& Bundesen, C. (2003). Clever homunculus: Is there an endogenous act of control in explicit task cuing procedures? Journal of Experimental Psychology: Human, Perception and Performance, 29, 575-599.

Lohmann, G., Mueller, K., Bosch, V., Mentzel, H., Hessler, S., Chen, L., Zysset, S., \& von Cramon, D. Y. (2001). Lipsia-A new software system for the evaluation of functional magnetic resonance images of the human brain. Computing Medical Imaging Graph, 25, 449-457.

Luks, T. L., Simpson, G. V., Feiwell, R. J., \& Miller, W. J. (2002). Evidence for anterior cingulate cortex involvement in monitoring preparatory attentional set. Neuroimage, 17, 792-802.

MacDonald, A. W., III, Cohen, J. D., Stenger, V. A., \& Carter, C. S. (2000). Dissociating the role of the dorsolateral prefrontal and anterior cingulate cortex in cognitive control Science, 288, 1835-1838.

Mayr, U., \& Kliegl, R. (2000). Task-set switching and long-term memory retrieval. Journal of Experimental Psychology: Learning, Memory, and Cognition, 26, 1124-1140.

Mayr, U., \& Kliegl, R. (2003). Differential effects of cue changes and task changes on task-set selection costs. Journal of Experimental Psychology: Learning, Memory, Cognition, 29, 362-372.

Meiran, N. (1996). Reconfiguration of processing mode prior to task performance. Journal of Experimental Psychology: Learning, Memory and Cognition, 22, 1423-1442.

Miller, E. K., \& Cohen, J. D. (2001). An integrative theory of prefrontal cortex function. Annual Review of Neuroscience, 24, 167-202.

Milner, B. (1963). Effects of different brain lesions on card sorting. Archives of Neurology, 9, 90-100.

Monchi, O., Petrides, M., Petre, V., Worsley, K., \& Dagher, A. (2001). Wisconsin Card Sorting revisited: Distinct neural circuits participating in different stages of the task identified by event-related functional magnetic resonance imaging. Journal of Neuroscience, 21, 7733-7741.

Nagahama, Y., Okada, T., Katsumi, Y., Hayashi, T., Yamauchi, H., Oyanagi, C., Konishi, J., Fukuyama, H., \& Shibasaki, H. (2001). Dissociable mechanisms of attentional control within the human prefrontal cortex. Cerebral Cortex, 11, 85-92.

Nakahara, K., Hayashi, T., Konishi, S., \& Miyashita, Y. (2002). Functional MRI of macaque monkeys performing a cognitive set-shifting task. Science, 295, 1532-1536.

Oldfield, R. C. (1971). The assessment and analysis of handedness: The Edinburgh Inventory.

Neuropsychologia, 9, 97-113.

Petrides, M., \& Pandya, D. N. (1999). Dorsolateral prefrontal cortex: Comparative cytoarchitectonic analysis in the human and the macaque brain and corticocortical connection patterns. European Journal of Neuroscience, 11, 1011-1036.

Pollmann, S., Dove, A., von Cramon, D. Y., \& Wiggins, C. J. (2000). Event-related fMRI: Comparison of conditions with varying BOLD overlap. Human Brain Mapping, 9, 26-37.

Rao, H., Zhou, T., Zhou, Y., Fan, S., \& Chen, L. (2003). Spatiotemporal activation of the two visual pathways in form discrimination and spatial location: A brain mapping study. Human Brain Mapping, 18, 79-89.
Rogers, R. D., \& Monsell, S. (1995). Costs of a predictable switch between simple cognitive tasks. Journal of Experimental Psychology: General, 124, 207-231.

Rubinstein, J. S., Meyer, D. E., \& Evans, J. E. (2001). Executive control of cognitive processes. Journal of Experimental Psychology: Human Perception and Performance, 27, 763-797.

Ruge, H., Brass, M., Koch, I., Rubin, O., Meiran, N., \& von Cramon, D. Y. (submitted). Reconciling advance preparation with proactive interference in task switching: Insights from BOLD fMRI.

Rushworth, M. F. S., Hadland, K. A., Paus, T., \& Sipila, P. K. (2001). Role of the human medial frontal cortex in task switching. A combined fMRI and TMS study. Journal of Neuroscience, 87, 2577-2592.

Rushworth, M. F. S., \& Owen A. M. (1998). The functional organization of the lateral frontal cortex: Conjecture or conjuncture in the electrophysiology literature? Trends in Cognitive Sciences, 2, 46-53.

Rushworth, M. F. S., Paus, T., \& Sipila, P. K. (2001). Attention systems and the organization of the human parietal cortex Journal of Neuroscience, 21, 5262-5271.

Schubotz, R. I., \& von Cramon, D. Y. (2002). Dynamic patterns make the premotor cortex interested in objects: Influence stimulus and task revealed by fMRI. Cognitive Brain Research, 14, 357-369.

Schumacher, E. H., \& D'Esposito, M. (2002). Neural implementation of response selection in humans as revealed by localized effects of stimulus-response compatibility on brain activation. Human Brain Mapping, 17, 193-201.

Sohn, M. H., Ursu, S., Anderson, J. R., Stenger, V. A., \& Carter, C. S. (2000). The role of prefrontal cortex and posterior parietal cortex in task switching. Proceedings of the National Academy of Sciences, U.S.A., 97, 13448-13453.

Sudevan, P., \& Taylor, D. A. (1987). The cueing and priming of cognitive operations. Journal of Experimental Psychology: Human Perception and Performance, 13, 89-103.

Talairach, P., \& Tournoux, J. (1988). A stereotactic coplanar atlas of the buman brain. Stuttgart: Thieme.

Tomita, H., Ohbayashi, M., Nakahara, k., Hasegawa, I., \& Mijashita, Y. (1999). Top-down signals from prefrontal cortex in executive control of memory. Nature, 401, 699-703.

Toni, I., Rushworth, M. F. S., \& Passingham, R. E. (2001). Neural correlates of visuomotor associations-Spatial rules compared with arbitrary rules. Experimental Brain Research, 141, 359-369.

Ullsperger, M., \& von Cramon, D. Y. (2001). Subprocesses of performance monitoring: A dissociation of error processing and response competition revealed by event-related fMRI and ERPs. Neuroimage, 14, 1387-1401.

Wise, S. P., \& Murray, E. A. (2000). Arbitrary associations between antecedents and actions. Trends Neuroscience, 23, 271-276.

Wallis, J. D, Anderson, K. C., \& Miller, E. K. (2001). Single neurons in prefrontal cortex encode abstract rules. Nature, 411, 953-956. 